Math. Model. Nat. Phenom.

Vol. 4, No. 2, 2009, pp. 119-139

DOI: $10.1051 / \mathrm{mmnp} / 20094206$

\title{
New Computational Tools for Modeling Chronic Myelogenous Leukemia
}

\author{
M. M. Peet ${ }^{1}{ }^{*}$, P. S. Kim ${ }^{2}$, S.-I. Niculescu ${ }^{3}$ and D. Levy ${ }^{4}$ \\ ${ }^{1}$ Illinois Institute of Technology, Chicago, USA. \\ ${ }^{2}$ Department of Mathematics, University of Utah, Salt Lake City 84102, USA. \\ ${ }^{3}$ Laboratoire des Signaux et Systèmes, CNRS-Supélec, 91192 Gif-sur-Yvette, France. \\ ${ }^{4}$ Department of Mathematics and Center for Scientific Computation and Mathematical Modeling, \\ University of Maryland, College Park 20742, USA
}

\begin{abstract}
In this paper, we consider a system of nonlinear delay-differential equations (DDEs) which models the dynamics of the interaction between chronic myelogenous leukemia (CML), imatinib, and the anti-leukemia immune response. Because of the chaotic nature of the dynamics and the sparse nature of experimental data, we look for ways to use computation to analyze the model without employing direct numerical simulation. In particular, we develop several tools using Lyapunov-Krasovskii analysis that allow us to test the robustness of the model with respect to uncertainty in patient parameters. The methods developed in this paper are applied to understanding which model parameters primarily affect the dynamics of the anti-leukemia immune response during imatinib treatment. The goal of this research is to aid the development of more efficient modeling approaches and more effective treatment strategies in cancer therapy.
\end{abstract}

Key words: delay-differential equations, model verification, optimization, polynomials, sum-ofsquares, stability, Lyapunov-Krasovskii, chronic myelogenous leukemia, imatinib

AMS subject classification: 34D10, 34K60, 92-08, 92C50

*Corresponding author. E-mail: mpeet@iit.edu 


\section{Introduction}

The representation of a physical system by mathematical formalism requires not only an intimate knowledge of the behavior of the natural phenomenon, but also a broad understanding of when and how mathematical equations can be used. An accurate model of a physical system, once created, can serve many purposes well beyond the scope or vision of the creator. In the ideal case, a model will faithfully represent the physical system whether the application be in control or prediction, at the macro-level or at the micro-level, interacting or in isolation. Of course, such a model will tend to grow in complexity to match the complexity of the physical system. In practice, models are often made ad-hoc, with a specific application in mind.

In this paper, we study the effects of chronic myelogenous leukemia (CML) using a delayed response model of the dynamics of the anti-leukemia immune response during Gleevec treatment. As presented in [14], the model is a state-based model in which cells transfer between various states based on stimuli or natural rates of cell development. The model describes the dynamics of two competing populations: leukemia cells and anti-leukemia T cells. A diagram of the dynamics of the model is shown in Figure 1.

The question of how to verify or improve a mathematical representation is a difficult one. To some extent, the process of refinement will be dictated by the use to which the model will be put. This is a reflection of the fact that models are often not completely accurate, but rather tools which are designed with a specific purpose in mind. In the vast majority of cases, the method of verification has been simulation and comparison with experimental data. There are several disadvantages to this approach in the case of myelogenous leukemia.

- The dynamics of the model are nonlinear and infinite-dimensional. Arbitrarily small errors in the initial condition may grow exponentially with time.

- The available experimental data are extremely sparse. Typical subjects will only have one or two measurements of the cancer cell population

- The data collected is insufficient to reconstruct the state of the system. This is a common problem in modeling infinite-dimensional systems such as those with time-delay. In addition, cancer cell populations are not measured. Only anti-cancer T-cells are measured.

- The measurements are made over large time-scales. Typically several years.

- The measurement error is large.

When these factors are present, simulation may be a poor predictor of model performance.

For the case of chronic myelogenous leukemia, we must also consider the purpose of the model. This particular model was developed to identify the immune system response. The goal of quantifying this response is to allow for the development of more effective treatments which make use of this phenomenon. Therefore, while the development of effective simulations is helpful, in that it allows one to test treatment strategies in silica, the question of how the model can assist in developing treatment strategies is not addressed. 
In this paper, we use robust control theory to address directly the question of whether the model accurately represents nature given uncertainties in measurement and patient data. We use newly developed computational methods to give model-based predictions when the patient data lies in some bounded region. The results are then compared with patient data to obtain areas of agreement.

The paper is organized as follows. In Section 2, we discuss the physiological background and present the model. In Section 3, we discuss computational methods of analysis. In Section 4, we consider the robustness of the model to patient parameters. In particular, in Section 4.1, we consider the question of the effect of delay on the stability of the model. Finally, in Section 5, we present concluding remarks.

\section{Biological background}

Chronic myelogenous leukemia (CML) is a cancer of the blood and bone marrow associated with the genetic mutation commonly known as the Philadelphia chromosome. The Philadelphia chromosome refers to a reciprocal translocation between chromosomes 9 and 22, which can be detected in more than $90 \%$ of all patients with CML [44]. The mutation results in the combination of two genes, the tyrosine kinase ABL gene of chromosome 9 and the BCR gene of chromosome 22, forming the BCR-ABL fusion gene [44]. The BCR-ABL gene results in continuously active transcription of the enzyme abl tyrosine kinase, causing the affected cell to keep dividing independently of other signals [39].

Currently, the standard therapy against CML is a molecular targeted drug called imatinib. Imatinib works by blocking the active site of the abl tyrosine kinase enzyme to inhibit uncontrolled division [3]. Under imatinib treatment, nearly all patients achieve hematologic remission [20] and $75 \%$ achieve cytogenetic remission [12]. However, imatinib does not completely eliminate leukemia cells and patients still have detectable disease at low levels [33]. In addition, patients inevitably relapse after stopping treatment [12].

Apart from imatinib, there is also increasing evidence that the immune system plays a key role in controlling CML and even sustaining imatinib-induced remission [2, 5, 8, 22, 26, 40, 44]. These recent studies indicate that a patient's own immune system can mount a response against leukemia cells. However, it is well-known that allogeneic bone-marrow or stem cell transplantation (ABMT or ASCT) can completely cure CML via a blood-restricted graft-versus-host immune response mediated by donor lymphocytes $[19,41,45]$. In fact, ABMT is currently the only known cure for CML [41]. Furthermore, infusion of allogeneic donor lymphocytes after transplantation induces complete cytogenetic response (CR) in $75 \%$ of CML patients who relapse after ABMT $[11,16]$. The graft-versus-host response that eliminates CML is primarily mediated by $\mathrm{T}$ cells, specifically CD8+ cells [15]. Hence, the adaptive immune response, particularly cytotoxic CD8+ T cell activity, plays an important role in controlling and possibly eliminating leukemia cells.

Without treatment, CML typically progresses through three phases. The first is the chronic phase, in which the cancer grows relatively slowly, the patient shows little or no outward symptoms, and the disease is responsive to therapy. Imatinib generally works most effectively during 
the chronic phase and can maintain cytogenetic remission of leukemia for five years or more [38]. However, due to acquired resistance mutations to the drug, the chronic phase is eventually followed by a brief accelerated phase, during which the disease accelerates growth and becomes less responsive to therapy. After the accelerated phase, the final phase is called the blast crisis. During blast crisis, the disease no longer responds well to other therapeutic interventions, except possibly ABMT, which is mediated by an anti-leumkemia immune response.

\subsection{Modeling background}

In this paper, we seek to model a patient's anti-leukemia immune response as a delay differential equation. Delay differential equation (DDE) models have been used in the past for immune system modeling [4], but not as often as the more prevalent ordinary differential equations (ODEs) [36]. Other alternative approaches include stage-based and agent-based modeling [7], each of which offer different trade-offs in complexity and biological comprehensiveness. For immune modeling, DDE systems offer a unique advantage, because they provide a means for incorporating programmed immune responses. Several experimental studies show that when stimulated by a target, T cells undergo a program of division even if the original stimulation is removed [13, 24, 46]. Thus, the overall immune response at a given time is not directly dependent upon the current level of antigenic stimulus, but on the stimulus at some time in the past. This delayed dependence can be seen experimentally, since the $T$ cell peak can occur even after complete clearance of antigen [28].

Among the DDE models that have been formulated to investigate immune and cancer dynamics are models by Luzyanina et al. who used a delayed system to describe T cell interactions with lymphocytic choriomeningitis virus [21]; Nelson and Perelson who devised a delay model to examine the influence of antiretroviral drugs on HIV [30]; and Villasana and Radunskaya who employed DDEs in a model considering tumor cells, immune cells, and a drug interfering with a specific phase of the cell cycle [47]. The delays in these models account for the transition times between various stages of immune and general cellular development, such as the progression from naïve to effector $\mathrm{T}$ cells, uninfected to infected T cells, and interphase to mitosis.

Two models that specifically study the immune response to CML are [29] and [27]. In the first work, Neiman formulates a DDE model to explain the transition of leukemia from the stable chronic phase to the unstable accelerated and acute phases [29]. In the second work, Moore and Li devise an ODE model and examine which model parameters are the most important in the success or failure of cancer remission. They conclude that cancer growth and death rates are the most important, and specifically that lower growth rates lead to a greater chance of cancer elimination [27].

Several recent models of CML have also been developed that do not incorporate the antileukemia immune response. For example, in [25], Michor et al. formulate a model for the dynamics of CML under imatinib treatment. Their model focuses, in particular, on the various levels of development of CML cells from the stem cell to the fully differentiated stage. In their model, imatinib greatly hinders the rate of progression through the various levels of development, causing a sudden drop in the leukemia population that happens in two distinct phases. They propose that 
imatinib cannot affect CML stem cells, allowing the disease to inevitably persist. This model is formulated as a system of linear ODEs, and in our paper, we use this model and incorporate an anti-leukemia immune response.

In a related but independent paper, Roeder et al. formulate an alternative model of CML and imatinib in which CML stem cells exist in either quiescent or proliferating phases [38]. Unlike the Michor model, the Roeder model assumes imatinib affects proliferating stem cells. Since proliferating stem cells can circulate back into the quiescent phase, imatinib can thus eventually affect all stem cells. This alternative hypothesis leads to different CML dynamics involving the strength of imatinib treatment and the predicted duration of CML remission. Furthermore, it suggests a treatment strategy that could work by accelerating the rate at which quiescent CML stem cells become affected by imatinib. Their model is formulated as a stochastic agent-based model to include the effects of probabilistic effects and discrete cell populations.

Another approach comes from Komorova and Wodarz who develop a model that focuses on the drug resistance of CML cells [17]. In their model, they also assume that imatinib can influence all CML cells including stem cells and that relapse results primarily from acquired imatinib resistance mutations, rather than a steady growth of the stem cell compartment as in the Michor model [17, 25]. Komorova and Wodarz propose a treatment strategy involving multiple CML-targeted drugs to reduce the probability of any leukemia cell eventually acquiring resistance-mutations to all drugs. In particular, they use their model to mathematically determine that a treatment strategy of three drugs of relatively uncorrelated activity has a high chance of curing the disease. The difficulty with the treatment lies, however, in the fact that it is currently unclear what alternative drugs to imatinib could be used for a multiple-drug strategy as such treatments have not been developed yet. Their model is formulated as a discrete-time, stochastic model that updates cell populations at every time step based on various probabilistic processes. Essentially, it is similar in framework to the agent-based model of Roeder et al. .

Several other age-structured and maturity-structured models for cell differentiation have been formulated as systems of PDEs, For example, Colijn and Mackey $[9,10]$ and Pujo-Menjouet and Mackey provide two age-structured models for normal hematopoiesis and for periodic CML [37]. In these models, cells spend constant amounts of time in various stages of development before progressing to successive stages. The models are formulated as systems of delay-differential equations (DDEs), where the delay values correspond to the time duration of each stage of cell development. As an alternative approach, Adimy and Pujo-Menjouet propose a model of cell division, in which the duration of each round of proliferation depends on the maturity of the cell [1]. This model is formulated as a system of hyperbolic PDEs with age and maturity variables that both increase over time. 


\subsection{The model}

Our model is illustrated in the state diagram contained in Figure 1.

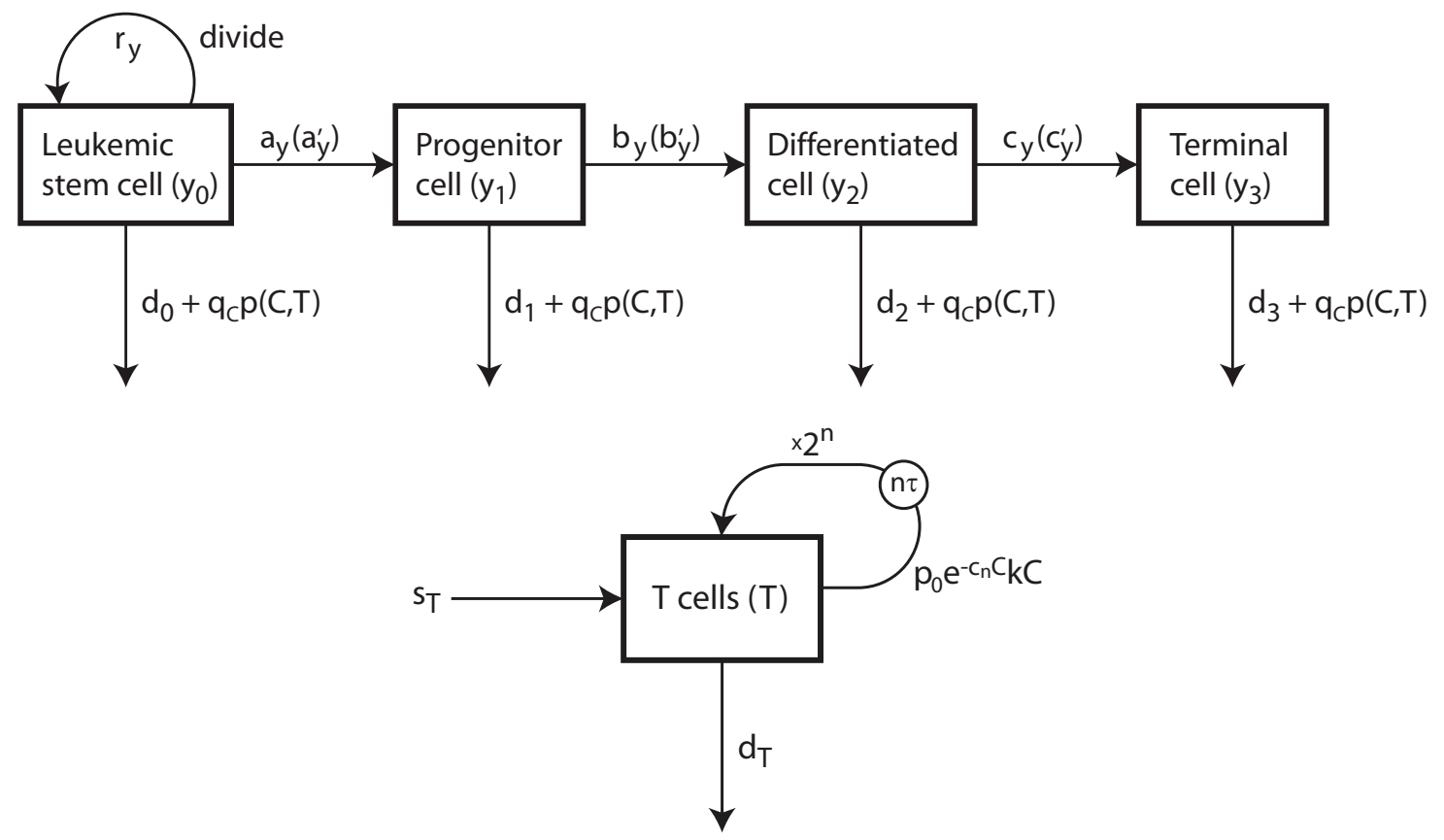

Figure 1: State diagrams for the dynamics of cancer and T cells in the model developed in [14]. The system of DDEs for the model is shown in (2.1).

The model consists of the five equations given below:

$$
\begin{aligned}
\dot{y}_{0}(t) & =\left[r_{y}-d_{0}\right] y_{0}(t)-q_{C} p(C(t), T(t)) y_{0}(t), \\
\dot{y}_{1}(t) & =a_{y} y_{0}(t)-d_{1} y_{1}(t)-q_{C} p(C(t), T(t)) y_{1}(t), \\
\dot{y}_{2}(t) & =b_{y} y_{1}(t)-d_{2} y_{2}(t)-q_{C} p(C(t), T(t)) y_{2}(t), \\
\dot{y}_{3}(t) & =c_{y} y_{2}(t)-d_{3} y_{3}(t)-q_{C} p(C(t), T(t)) y_{3}(t), \\
\dot{T}(t) & =s_{T}-d_{T} T(t)-p(C(t), T(t)) C(t) \\
& +2^{n} p(C(t-n \tau), T(t-n \tau)) q_{T} C(t-n \tau),
\end{aligned}
$$

where

$$
p(C, T)=p_{0} e^{-\gamma C} k T, \quad C=y_{0}+y_{1}+y_{2}+y_{3} .
$$

The first four equations are based on the model in [25]. They govern the dynamics of leukemia cells, and the variables $y_{0}, y_{1}, y_{2}$, and $y_{3}$ denote the concentrations of leukemia stem cells, progenitor cells, differentiated cells, and terminally differentiated cells. In addition, the parameters $r_{y}$, $a_{y}, b_{y}, c_{y}$, and $d_{i}$ denote the rates of growth, differentation, and death of each of the four stages of leukemia cell differentiation. The fifth equation as well as the final terms in the first four equations 
pertain to the dynamics of anti-leukemia $\mathrm{T}$ cells and interactions between $\mathrm{T}$ cells and leukemia cells. The variable $T$ denotes the concentration of $T$ cells, and the time delay, $n \tau$, corresponds to the duration of $\mathrm{T}$ cell division. A thorough discussion of these equations and parameters is presented in [31].

In the original presentation and analysis of the model in [31], Niculescu et al. conduct a linear stability analysis for three parameter sets corresponding to three patients. In their analysis, they exploit the particular rank one structure of the delay matrix and use a geometric argument to find that the system is robustly stable for all three parameter sets for a wide range of delay values.

In [23], Mazenc et al. assume Gleevec is slightly less effective than estimated in [25] and update the death rate parameters appropriately. Using the new parameters, linear stability analysis yields that the system is robustly stable for two out of three of the parameter sets. However, the system is unstable for one of the parameter sets. In this paper, Mazenc et al. continue with a global nonlinear analysis in which they perform a coordinate transformation to isolate a linear ordinary differential system from an auxiliary nonlinear delay differential equation system. In this manner, they reduce the original five-dimensional nonlinear problem to the analysis of a two-dimensional nonlinear system and prove the existence of unbounded trajectories. Furthermore, they present sufficient conditions for the initial leukemia and $\mathrm{T}$ cell concentrations that guarantee unbounded solutions.

In this paper, we are interested in examining how parameters other than the time delay or initial conditions affect stability. The main difficulty with this problem is that perturbing any of these parameters changes the equilibrium cell concentrations, which in turn affects stability properties. Hence, to conduct this analysis, we need to consider Lyapunov-Krasovskii functionals of the delay diferential equation (DDE) system under algebraic constraints, which come from the implicit equation for the equilibrium cell concentrations. This analysis is useful from a biological point of view, because we are interested in how the model is affected by a range of parameters beyond only the delay parameter and initial cell concentrations.

\section{Computational tools}

In this section, we describe an optimization-based approach to robust analysis of nonlinear and time-delayed systems.

\subsection{The positivstellensatz and sum-of-squares}

In this section, we present some recent results on optimization of polynomials which will be used in the computational analysis.

Notation: A polynomial $p$ is said to be positive on $G \subset \mathbb{R}^{n}$ if

$$
p(x) \geq 0 \quad \text { for all } x \in G \text {. }
$$


If $G$ is not mentioned, then it is assumed $G=\mathbb{R}^{n}$. A semialgebraic set is a subset of $\mathbb{R}^{n}$ defined by polynomials $p_{i}$, as

$$
G:=\left\{x \in \mathbb{R}^{n}: p_{i}(x) \geq 0, i=1, \ldots, k\right\} .
$$

A polynomial $p(x)$ is said to be sum-of-squares (SOS) in variables $x$, denoted $p \in \Sigma_{s}[x]$ if there exist a finite number of other polynomials $g_{i}$ such that

$$
p(x)=\sum_{i=1}^{k} g_{i}(x)^{2} .
$$

Lemma 1. A necessary and sufficient condition for the existence of a sum-of-squares representation for a polynomial $p$ of degree $2 d$ is the existence of a positive semidefinite matrix, $Q$, such that

$$
p(x)=Z(x)^{T} Q Z(x)
$$

where $Z$ is any vector whose elements form a basis for the polynomials of degree $d$.

Clearly, the existence of an SOS representation is sufficient for positivity of the polynomial $p$. However, it is not necessary. The advantage of working with the cone of SOS polynomials, as opposed to simply positive polynomials, is the fact that any SOS polynomial can be represented by a positive matrix. Thus positivity of a polynomial can be represented using a Linear Matrix Inequality (LMI). LMIs are a form of convex optimization over the positive matrices. There exist very efficient interior-point algorithms for optimization of positive matrices such as Sedumi [43] or DSPD [6]. Thus optimization of SOS polynomials is relatively routine. The issue of accuracy with respect to all positive polynomials can be addressed using Positivstellensatz-type results, as described below.

Definition 2. A set is semi-algebraic if it can be represented using polynomial inequality and equality constraints of the form

$$
\left\{x: p_{i}(x) \geq 0, q_{j}(x)=0, i, j=1, \cdots, N\right\}
$$

where the $p_{i}$ and $q_{j}$ are polynomial.

Semi-algebraic sets are important in polynomial optimization in that they offer refutations of positivity, e.g. $p(x)>0$ for all $x$ if and only if $\{x:-p(x) \geq 0\}=\emptyset$. Thus feasibility of a semi-algebraic set is an alternative to polynomial positivity.

Positivstellensatz results are "theorems of the alternative" which say that either a semialgebraic set is feasible or there exists a sum-of-squares refutation of feasibility. The Positivstellensatz that we use in this paper is that given by Stengle [42].

Theorem 3 (Stengle). The following are equivalent

1.

$$
\left\{x: \begin{array}{ll}
p_{i}(x) \geq 0 & i=1, \ldots, k \\
q_{j}(x)=0 & j=1, \ldots, m
\end{array}\right\}=\emptyset
$$


2. There exist $t_{i} \in \mathbb{R}[x], s_{i}, r_{i j}, \ldots \in \Sigma_{s}[x]$ such that

$$
-1=\sum_{i} q_{i} t_{i}+s_{0}+\sum_{i} s_{i} p_{i}+\sum_{i \neq j} r_{i j} p_{i} p_{j}+\cdots
$$

We use $\mathbb{R}[x]$ to denote the real-valued polynomials in variables $x$. For a given degree bound, the conditions associated with Stengle's Positivstellensatz can be represented as a semidefinite program. Note that, in general, no such upper bound on the degree bound will be known a-priori. Positivstellensatz results can be used to prove robust stability of uncertain systems or local stability of nonlinear or chaotic systems.

\subsection{Stability analysis using polynomials}

Consider an ordinary differential equations of the form

$$
\dot{x}(t)=f(x(t))
$$

where $f: \mathbb{R}^{n} \rightarrow \mathbb{R}^{n}$ is continuous. This system is exponentially stable if there exists a differentiable function $V: \mathbb{R}^{n} \rightarrow \mathbb{R}$ such that there exist $\alpha, \beta, \gamma>0$ such that

$$
\begin{array}{r}
\alpha\|x\|^{2} \leq V(x) \leq \beta\|x\|^{2} \\
\dot{V}(x)=\nabla V(x)^{T} f(x) \leq-\gamma\|x\|^{2}
\end{array}
$$

for any $x$ along trajectories of the system. If the conditions only hold on a subset $\Omega \subset \mathbb{R}^{n}$, then the region of attraction of the trivial equilibrium can be estimated as $Y_{\delta}:=\{x: v(x) \leq \delta\} \subset \Omega$ for any $\delta>0$.

For linear systems, quadratic Lyapunov functions are necessary and sufficient for stability. This means that stability analysis of state-space systems can be done algorithmically using Linear Matrix Inequalities (LMIs). The following Proposition shows how this is done for robust stability conditions for linear systems with parametric uncertainty.

Proposition 4. Suppose there exists a polynomial $A: \mathbb{R}^{n} \times \mathbb{R}^{m} \rightarrow \mathbb{R}$, a constant $\varepsilon>0$, and sum-of-squares polynomials $S_{i}, T_{i}: \mathbb{R}^{n} \times \mathbb{R}^{m} \rightarrow \mathbb{R}$ such that

$$
P(\alpha)-\sum_{i} S_{i}(\alpha) q_{i}(\alpha) \geq \varepsilon I
$$

and

$$
A(\alpha)^{T} P(\alpha)+P(\alpha) A(\alpha)+\sum_{i} T_{i}(\alpha) q_{i}(\alpha) \leq-\varepsilon I
$$

Then $\dot{x}(t)=A(\alpha) x(t)$ is exponentially stable for any fixed $\alpha$ such that $q_{i}(\alpha) \geq 0$.

That is, the system is stable when the parameter $\alpha$ lies in the semialgebraic set defined by the $q_{i}$. In the same manner, Positivstellensatz results can be used to derive local stability conditions for nonlinear ordinary differential equations. 
Proposition 5. Suppose there exists a polynomial $V: \mathbb{R}^{n} \rightarrow \mathbb{R}$, a constant $\varepsilon>0$, and sum-ofsquares polynomials $s_{i}, t_{i}: \mathbb{R}^{n} \rightarrow \mathbb{R}$ such that

$$
V(x)-\sum_{i} s_{i}(x) q_{i}(x) \geq \varepsilon x^{T} x
$$

and

$$
\nabla V(x)^{T} f(x)+\sum_{i} t_{i}(x) q_{i}(x) \leq-\varepsilon x^{T} x
$$

Then there exist constants $\mu, \delta, r>0$ such that

$$
\|x(t)\|_{2} \leq \mu\left\|x_{0}\right\|_{2} e^{-\delta t}
$$

for all $t \geq 0$ and initial conditions $x_{0} \in Y_{\delta}:=\{x: v(x) \leq \delta\} \subset Q$, where $Q:=\left\{x: q_{i}(x) \geq 0\right\}$ and $\delta>0$.

That is, $f$ is stable on the largest level set of $V$ contained in the semialgebraic set defined by the $q_{i}$.

\subsection{Systems with time delay}

Time delays, while a common modeling tool, can be very difficult to account for in analysis. Much research has been devoted to this subject and a number of computational tools have been proposed. We concentrate on the advances related to Lyapunov analysis. First consider a system with a time-delay of the following form.

$$
\dot{x}(t)=f(x(t), x(t-\tau)),
$$

where $f: \mathbb{R}^{n} \times \mathbb{R}^{n} \rightarrow \mathbb{R}^{n}$ is continuous and $\tau \geq 0$. For these systems, the state is infinitedimensional and Lyapunov theory must be generalized [18] using Lyapunov-Krasovskii functions. Now the theory states the system is stable if one can find a Lyapunov-Krasovskii function $V$ : $\mathcal{C}[-\tau, 0] \rightarrow \mathbb{R}$ and positive constants $\alpha, \beta, \gamma$ such that

$$
\|\phi(0)\|^{2} \leq V(\phi) \leq \beta\|\phi\|_{\infty}^{2}
$$

and

$$
\dot{V}(\phi) \leq-\gamma\|\phi(0)\|^{2}
$$

hold for any segment of trajectory, $\phi$, and where $\dot{V}(\phi)$ is the derivative of $V$ along trajectories of the system. Note that the function, $V$, has an argument in the infinite-dimensional state-space $\mathcal{C}[-\tau, 0]$. The search for a Lyapunov function in this case is significantly more difficult than for finite-dimensional systems. If we consider only linear systems, we can refine the search to quadratic functions of the following form.

$$
\begin{aligned}
V(\phi)=\int_{-\tau}^{0}\left[\begin{array}{l}
\phi(0) \\
\phi(s)
\end{array}\right] & M(s)\left[\begin{array}{l}
\phi(0) \\
\phi(s)
\end{array}\right] d s \\
& +\int_{-\tau}^{0} \int_{-\tau}^{0} \phi(s) N(s, t) \phi(t) d s d t
\end{aligned}
$$


Conditions for stability of linear time-delay systems can be given using polynomial optimization. For simplicity, we only present the case of a single delay. See the references [35] or [32] for further explanation of these results and the generalization to multiple delays and nonlinear systems.

Theorem 6. The system $\dot{x}(t)=A_{0} x(t)+A_{1} x(t-\tau)$ is stable if there exist polynomials $M, T, N$ and positive semidefinite matrices $Q, R \geq 0$ such that the following conditions hold

$$
\begin{aligned}
M(s)+\left[\begin{array}{cc}
T(s) & 0 \\
0 & 0
\end{array}\right] & >0 \quad \text { for all } s \in[-h, 0], \\
\int_{-h}^{0} T(s) d s & =0, \\
N(s, t) & =Z(s)^{T} Q Z(t), \\
-L(M, N)(s)+\left[\begin{array}{cr}
Q(s) & 0 \\
0 & 0
\end{array}\right] & >0 \text { for all } s \in[-h, 0], \\
\int_{-h}^{0} Q(s) d s & =0 . \\
\frac{\partial}{\partial s} N(s, t)+\frac{\partial}{\partial t} N(s, t) & =Z(s)^{T} R Z(t) .
\end{aligned}
$$

Here

$$
\begin{aligned}
& L(M, N)(s)=\left[\begin{array}{ccc}
A_{0}^{T} M_{11}+M_{11} A_{0} & M_{11} A_{1} & 0 \\
*^{T} & 0 & 0 \\
*^{T} & *^{T} & 0
\end{array}\right] \\
& +\left[\begin{array}{ccc}
0 & 0 & A_{0}^{T} M_{12}(s) \\
*^{T} & 0 & A_{1}^{T} M_{12}(s) \\
*^{T} & *^{T} & 0
\end{array}\right] \\
& +\frac{1}{h}\left[\begin{array}{cccc}
M_{12}(0)+M_{21}(0)+M_{22}(0) & -M_{12}(-h) & 0 \\
& *^{T} & -M_{22}(-h) & 0 \\
*^{T} & *^{T} & 0
\end{array}\right] \\
& +\left[\begin{array}{ccc}
0 & 0 & N(0, t)-\dot{M}_{12}(s) \\
*^{T} & 0 & -N(-h, t) \\
*^{T} & *^{T} & -\dot{M}_{22}(s)
\end{array}\right] .
\end{aligned}
$$

In this paper, we combine the results of Theorem 6 with the positivstellensatz to obtain robust stability conditions for time-delay systems. This mirrors the approach which was explained earlier in this section with respect to Proposition 4. In what follows, we show how these results are used to improve models of chronic myelogenous leukemia. 


\section{Analysis of the model}

\begin{tabular}{|l|l|c|c|c|}
\hline & Description (units) & $\mathrm{P} 1$ & $\mathrm{P} 4$ & $\mathrm{P} 12$ \\
\hline \hline$n$ & Average \# of T cell divisions & 2.2 & 1.2 & 1.17 \\
$d_{T}$ & T cell death rate & $1 \mathrm{E}-3$ & $2.2 \mathrm{E}-3$ & $7 \mathrm{E}-3$ \\
$s_{T}$ & T cell supply rate & $2 \mathrm{E}-6$ & $9 \mathrm{E}-7$ & $3 \mathrm{E}-5$ \\
$\gamma$ & Decay rate of immune response & 1 & 7 & 0.8 \\
\hline
\end{tabular}

Table 1: Definition of parameters and patient estimates

Consider the model given by $(2.1)$ as developed in [14, 31]. In this section we look at the problem of uncertainty in the values of the patient parameters. We first note that these parameters cannot, at present, be measured directly in an efficient manner. Rather, the values of these parameters, for example as listed in Table 1, are inferred in an a-posteriori manner from the clinical data by tuning the values so that observed behavior will match the predicted patient response. The values listed in Table 1 produce the simulated results matched with experimental data illustrated in Figure 2. A discussion of the collection of the data and estimation of the parameters is given in the thesis work of P. Kim [14].

This approach to identification of patient parameters means that the inferred values of the parameters will have significant error. Furthermore, in most practical situations such as diagnosis and treatment, it is expected that the precise values of these parameters will never be known since insufficient data will be available to allow for patient-specific identification. Our conclusion, therefore, is that the parameters must be treated as unknowns which lie in some certain region of the parameter space. The regions proposed are chosen so as to encompass the estimated values given in Table 1.

The technical contribution of this paper is to provide a computational tool which can improve understanding of the influence of parametric uncertainty on the behavior the equilibrium corresponding to hematological remission. We will not focus on the question of global stability, but will rather consider linear stability of the hematological remission. This means we will not address the question of cancer elimination. The goal of this work is to create a way of validating the behavior by examining only the question of whether or not a patient remains in long term remission without a complete elimination. This will allow us to test validity of the model using only sparse data or qualitative observations.

\subsection{The delay parameter}

For completeness, we analyze the the influence of the delay parameter. Note, however, that a previous linear analysis of the delay parameter can be found in [23] and [31] using analytical and graphical methods respectively. Analysis of the delay is significantly simpler than for the other parameters and only a short outline of our procedure and results will be presented here. Particularly important for the delay parameter is that the location of the equilibrium point does not change with 


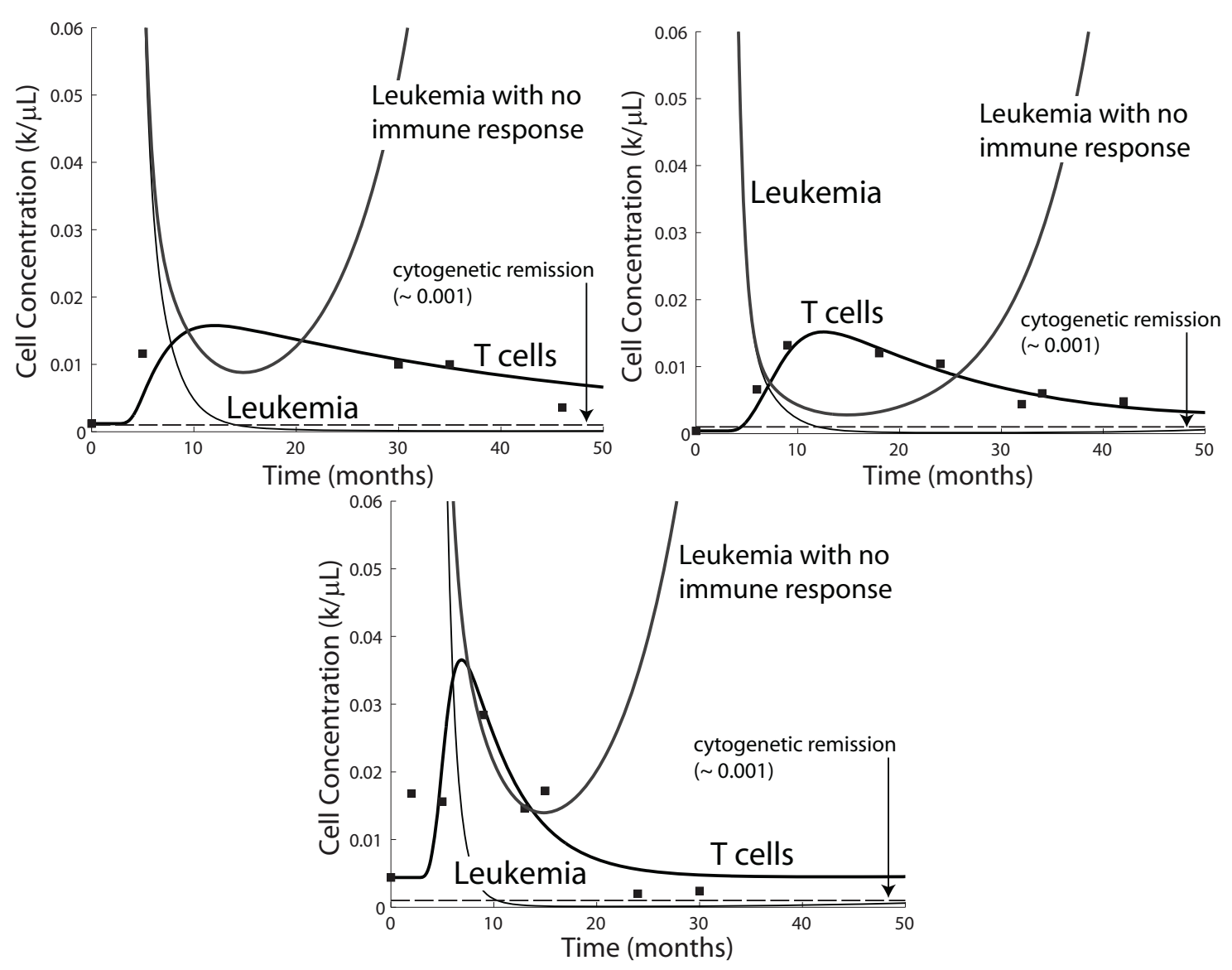

Figure 2: Data from (reading from upper left) patients 1, 4, and 12 compared to modeled response.

the value of the delay. Therefore, by using the values of the non-delay patient parameters, as given in Table 1, one can linearize the dynamics by taking the Jacobian at these values.

In our analysis, the results of which can be found in Table 2, for each set of patient data, we used the Lyapunov-Krasovskii LMI conditions associated with Theorem 6 combined with the robust analysis tools associated with Proposition 4 to construct a Lyapunov-Krasovskii functional valid over the range of delay values $\tau \in[0, \bar{\tau}]$. Because the automated procedure requires relatively little time, we were able to use a bisection method to determine the maximum $\bar{\tau}$, the values of which are listed in Table 2. These results strongly indicate that delay is not a sensitive parameter on short time-scales given reasonable values of the other patient parameters.

\subsection{Non-delay parameters}

In this subsection, we consider how to account for variation in several of the patient-specific parameters included in the model. As stated before, this paper does not directly address the problem of nonlinearity in the state. We are, however, interested in capturing the nonlinear effects of the parameter as it enters into the model. For this reason, we retain the nonlinear features of the model 


\begin{tabular}{|l|c|c|c|}
\hline Parameter & \multicolumn{3}{|c|}{ Estimates (P1, P4, P12) } \\
\hline \hline$n$ & 2.2 & 1.2 & 1.17 \\
$d_{T}$ & 0.001 & 0.0022 & 0.007 \\
$s_{T}$ & $2 \times 10^{-6}$ & $9 \times 10^{-7}$ & $3.08 \times 10^{-5}$ \\
$\gamma$ & 1 & 7 & 0.8 \\
\hline$\tau_{\max }$ & 222 & 190 & 334 \\
\hline
\end{tabular}

Table 2: Stability results for patients $\mathrm{P} 1, \mathrm{P} 4$, and $\mathrm{P} 12$.

while make some modifications which allow for more efficient computational testing.

Constructing a parameter-dependent model. Our first step in analyzing robustness of the model to parameter variation is to modify the model so as to be more amenable to computation. Specifically, at present the computational techniques described earlier can only be applied to dynamics described by polynomial or rational functions. Additionally, we would like to construct an approximation to the model which is linear in the state.

Since, as stated earlier, we are only interested in linear analysis, the first problem is easily overcome. All of the transcendental terms included in the original model may be approximated arbitrarily well by polynomials on a bounded region. Although there are many methods for making this approximation, we have chosen an interpolation-based method.

A more difficult problem is that the equilibrium point changes with variations in the parameters. For the delay parameter, our linear analysis used the value of the Jacobian at the equilibium point. Since the value of the equilibrium is not a closed-form function of the parameters, this approach is no longer applicable. Recall the nonlinear system (2.1). This system of equations has three equilibrium points for the range of parameter values we are interested in. To create a model which is linear in the state, but captures the nonlinear dependence on the parameters $n, d T, s T$, and $\gamma$, we calculate the Jacobian at regular values of the parameters and use an interpolation-based technique to construct an approximation to the parameter-dependent Jacobian. Although not truly rigorous, this method allows us to capture the essential behavior of the system. Some comments on the linearization procedure are as follows.

1. In order to simplify the presentation, we treat each variable separately. However, this is not necessary as one can just as easily interpolate in a multi-dimensional space.

2. The interpolation points are standard. See Figures 5 and 4.

3. Because the equilibrium point cannot be expressed as an explicit function of the system parameters, it must be solved numerically at each interpolation point using standard rootfinding algorithms. Finding the correct equilibrium point corresponding to hematological remission is relatively simple as it is several orders of magnitude removed from the others.

4. The interpolation method used least-squares error minimization. 
5. The interpolation error was constrained to be less than $.001 \%$ of the size of the range of values considered

While a number of elements of the system were not sensitive to variation of parameters, others showed variation. Some of this variation can be observed in the examples, as shown in the dependence of a particularly important element of the linear system matrices and plotted in Figure 3 . In most cases, we found that a second order polynomial was sufficient to provide the needed accuracy. The new parameter-dependent models are complex and thus are not fully included here for reasons of space. Figure 3 depicts only one of 50 elements of the model.
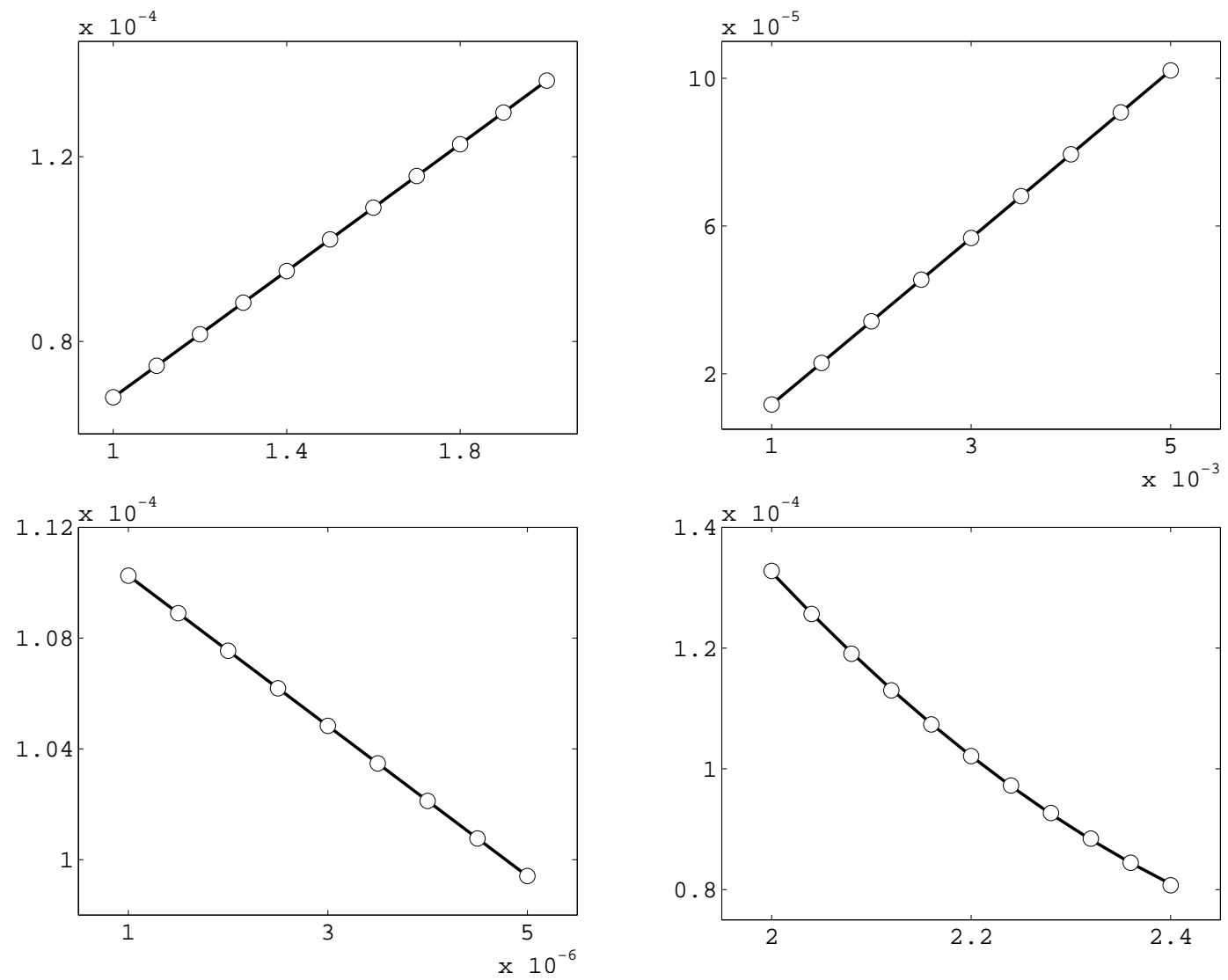

Figure 3: Variation in the 1,1 entry of $A_{0}$ as a function of, reading from upper left, $\gamma, d T, s T$, and $n$. Both the model and selected data points are shown.

Robustness analysis. Once a linear parameter-dependent model is obtained, the same methodology described in Section 4.1. can be applied. In particular, we use algorithms based on the LMIs in Theorem 6 combined with the robustness conditions of Proposition 4. General versions of these algorithms can be found online at [34].

Based on our analysis of the model with respect to the parameter, $\gamma$, we find that the system is stable for a relatively wide range of $\gamma$, the decay rate of immune responsitivity. This is illustrated 
in Figure 4 where we depict the maximum stable region (dashed line) along with certain estimated values from long-term stable patient data. We also include the expected stability range (solid line) for comparison. From a biological perspective, this would correlate well with the fact that the fixed point under consideration corresponds to a very low leukemia cell concentration, where leukemia cells are no longer effective at immune downregulation.

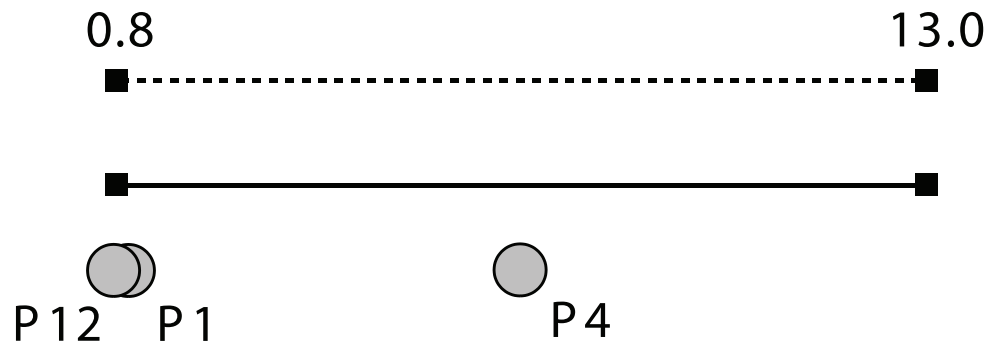

Figure 4: Stability region (dashed line) with respect to parameter $\gamma$ compared to patient data.

On the other hand, the system is more sensitive to the parameter $n$, the average number of $\mathrm{T}$ cell divisions after stimulation. This relationship is illustrated in Figure 5 where we depict the maximum stable region (dashed line) along with certain estimated values from long-term stable patient data. We also include the expected stability range (solid line) for comparison. Experimentally, $n$ corresponds to the level of T cell proliferation after stimulus. Hence, we conclude that the model predicts that effectiveness of treatment is highly associated with the latent responsitivity of anti-leukemia T cells before Gleevec-induced remission. This also corresponds well with observed behaviour. We also observe that the robust stability region does not include several values of the patient parameters. This is worrisome and suggests that either the values of the patient parameters are incorrect or modifications to the model may be needed to improve the robustness. It is noted, however, that the patient values are relatively close to the boundary. Therefore, the problem may in fact be due to simple numerical error.

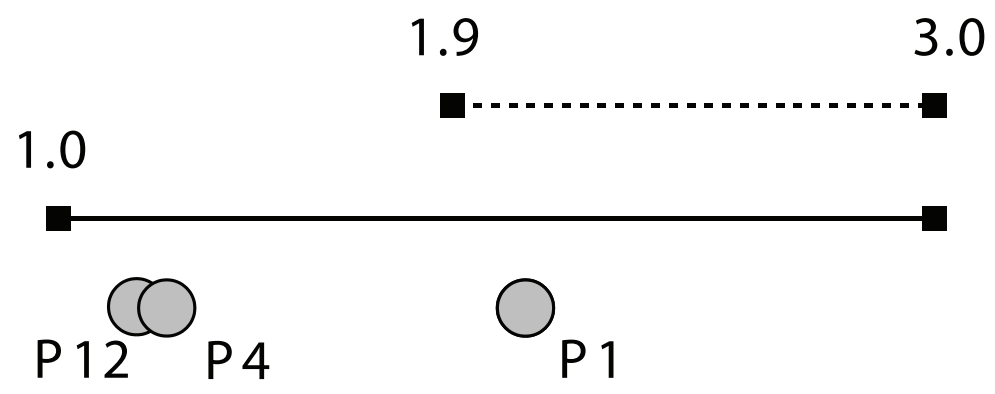

Figure 5: Stability region (dashed line) with respect to parameter $n$ compared to patient data.

Finally, we found that the model was not robustly stable with respect to $s_{T}$. This observation may imply that stability is highly sensitive to this parameter. If so, this finding corroborates the results of the nonlinear analysis of [23], in which Mazenc et al. conclude that low initial $\mathrm{T}$ cell 
concentrations coupled with high initial leukemia concentrations result in unbounded solutions. Since the initial T cell concentration is proportional to $s_{T}$, this interpretation is compatible with the findings of [23]. Further study is required to confirm this hypothesis.

\section{Concluding remarks}

In this paper, we pursue the use of new computational methods to improve a model of chronic myelogenous leukemia. As part of this process, we construct a linear parameter-dependent model of CML, develop algorithms for robust analysis of linear delayed systems, and compare our result with expected data. From our analysis, we conclude that the model predicts that stability of the system is sensitive to $\mathrm{T}$ cell-related parameters such as the responsitivity of $\mathrm{T}$ cells to stimulation and possibly the T cell supply rate into the system. This analysis suggests that the patient's latent immune response might play a nontrivial supporting role in maintaining cancer remission during Gleevec treatment.

\section{Acknowledgments}

The work of PSK was supported in part by the Chateaubriand Fellowship. The authors would like to express their thanks to Peter Lee, MD for his insight and guidance in formulating the mathematical model and for his feedback on relevant biological questions and implications.

\section{References}

[1] M. Adimy, L. Pujo-Menjouet. A mathematical model describing cellular division with a proliferating phase duration depending on the maturity of cells. Electronic Journal of Differential Equations, (2003) No. 107, 1-14.

[2] E.P. Alyea, R.J. Soiffer, C. Canning, D. Neuberg, R. Schlossman, C. Pickett, H. Collins, Y. Wang, K.C. Anderson, J. Ritz. Toxicity and efficacy of defined doses of CD4+ donor lymphocytes for treatment of relapse after allogeneic bone marrow transplant. Blood, 19 (1998), No. 10, 3671-3680.

[3] G.R. Angstreich, B.D. Smith, R.J. Jones. Treatment options for chronic myeloid leukemia: imatinib versus interferon versus allogeneic transplant. Curr. Opin. Oncol., 16 (2004), No. 2, 95-99.

[4] R. Antia, C.T. Bergstrom, S.S. Pilyugin, S.M. Kaech, R. Ahmed. Models of CD8+ responses: 1. What is the antigen-independent proliferation program. J. Theor. Biol., 221 (2003), No. 4, 585-598.

[5] A. Bagg. Chronic myeloid leukemia: a minimalistic view of post-therapeutic monitoring. J. Mol. Diagn., 4 (2002), No. 1, 1-10. 
[6] S.J. Benson, Y. Ye", DSDP5: Software For semidefinite programming. (Sept. 2005) Mathematics and Computer Science Division, Argonne National Laboratory, Argonne, IL, ANL/MCS-P1289-0905, http://www.mcs.anl.gov/ benson/dsdp, (Submitted to ACM Transactions on Mathematical Software).

[7] D.L. Chao, S. Forrest, M.P. Davenport, A.S. Perelson. Stochastic stage-structured modeling of the adaptive immune system. Proc. IEEE Comput. Soc. Bioinform. Conf., 2 (2003), 124131.

[8] C.I. Chen, H.T. Maecker, P.P. Lee. Development and dynamics of robust T-cell responses to CML under imatinib treatment. Blood, 111 (2008), No. 11, 5342-5349.

[9] C. Colijn, M.C. Mackey. A mathematical model of hematopoiesis-I. Periodic chronic myelogenous leukemia. J. Theor. Biol., 237 (2005), No. 2, 117-132.

[10] C. Colijn, M.C. Mackey. A mathematical model of hematopoiesis-II. Cyclical neutropenia. J. Theor. Biol., 237 (2005), No. 2, 133-146.

[11] R.H. Collins, Jr., O. Shpilberg, W.R. Drobyski, D.L. Porter, S. Giralt, R. Champlin, S.A. Goodman, S.N. Wolff, W. Hu, C. Verfaillie, A. List, W. Dalton, N. Ognoskie, A. Chetrit, J.H. Antin, J. Nemunaitis. Donor leukocyte infusions in 140 patients with relapsed malignancy after allogeneic bone marrow transplantation. J. Clin. Oncol., 15 (1997), No. 2, 433444.

[12] J. Cortes, M. Talpaz, S. O’Brien, D. Jones, R. Luthra, J. Shan, F. Giles, S. Faderl, S. Verstovsek, G. Garcia-Manero, M.B. Rios, H. Kantarjian. Molecular responses in patients with chronic myelogenous leukemia in chronic phase treated with imatinib mesylate. Clin. Cancer Res., 11 (2005), No. 9, 3425-3432.

[13] S.M. Kaech, R. Ahmed. Memory CD8+ T cell differentiation: initial antigen encounter triggers a developmental program in naïve cells. Nat. Immunol., 2 (2001), No. 5, 415-422.

[14] P.S. Kim. Mathematical Models of the Activation and Regulation of the Immune System. PhD thesis, Stanford University (2007).

[15] T. Klingebiel, P.G. Schlegel. GVHD: overview on pathophysiology, incidence, clinical and biological features. Bone Marrow Transplant., 21 (1998), Suppl. 2, S45-S49.

[16] H.J. Kolb, A. Schattenberg, J.M. Goldman, B. Hertenstein, N. Jacobsen, W. Arcese, P. Ljungman, A. Ferrant, L. Verdonck, D. Niederwieser, et al. Graft-versus-leukemia effect of donor lymphocyte transfusions in marrow grafted patients. European Group for Blood and Marrow Transplantation Working Party Chronic Leukemia". Blood, 86 (1995), No. 5, 2041-2050.

[17] N.L. Komarova, D. Wodarz. Drug resistance in cancer: Principles of emergence and prevention. Proc. Natl. Acad. Sci. USA, 102 (2005), No. 27, 9714-9719. 
[18] N.N. Krasovskii. Stability of Motion. Stanford University Press, 1963.

[19] K-A. Kreuzer, C.A. Schmidt, J. Schetelig, T.K. Held, C. Thiede, G. Ehninger, W. Siegert. Kinetics of stem cell engraftment and clearance of leukaemia cells after allogeneic stem cell transplantation with reduced intensity conditioning in chronic myeloid leukaemia. Eur. J. Haematol., 69 (2002), No. 1, 7-10.

[20] S.J Lee. Chronic myelogenous leukaemia. Br. J. Haematol., 111 (2000), No. 4, 993-1009.

[21] T. Luzyanina, K. Engelborghs, S. Ehl, P. Klenerman, G. Bocharov. Low level viral persistence after infection with LCMV: a quantitative insight through numerical bifurcation analysis. Math. Biosci., 173 (2004), No. 1, 1-23.

[22] W.A.E. Marijt, M.H.M. Heemskerk, F.M. Kloosterboer, E. Goulmy, M.G.D Kester, M.A.W.G. van der Hoorn, S.A.P. van Luxemburg-Heys, M. Hoogeboom, T. Mutis, J.W. Drijfhout, J.J. van Rood, R. Willemze, J.H.F. Falkenburg. Hematopoiesis-restricted minor histocompatibility antigens HA-1- or HA-2-specific T cells can induce complete remissions of relapsed leukemia. Proc. Natl. Acad. Sci. USA, 100 (2003), No. 5, 2742-2747.

[23] F. Mazenc, P.S. Kim, S.-I. Niculescu. Stability of a combined Gleevec and immune model involving delays: linear and global analysis. Proceedings of the 47th IEEE Conference on Decision and Control (2008).

[24] R. Mercado, S. Vijh, S.E. Allen, K. Kerksiek, I.M. Pilip, E.G. Pamer. Early programming of $T$ cell populations responding to bacterial infection. J. Immunol., 165 (2000), No. 12, 6833-6839.

[25] F. Michor, T.P. Hughes, Y. Iwasa, S. Branford, N.P. Shah, C.L. Sawyers, M.A. Nowak. Dynamics of chronic myeloid leukaemia. Nature, 435 (2005), No. 7046, 1267-1270.

[26] J.J. Molldrem, P.P. Lee, C. Wang, K. Felio, H.M. Kantarjian, R.E. Champlin, M.M. Davis. Evidence that specific T lymphocytes may participate in the elimination of chronic myelogenous leukemia. Nat. Med., 6 (2000), No. 8, 1018-1023.

[27] H. Moore, N.K. Li. A mathematical model for chronic myelogenous leukemia (CML) and T cell interaction. J. Theor. Biol., 225 (2004), No. 4, 513-523.

[28] K. Murali-Krishna, J.D. Altman, M. Suresh, D.J.D. Sourdive, D.J.D. Zajac, J.D. Miller, J. Slansky, R. Ahmed. Counting antigen-specific CD8+ T cells: a re-evaluation of bystander activation during viral infection. Immunity, 8 (1998), No. 2, 177-187.

[29] B. Neiman. A mathematical model of chronic myelogenous leukaemia. Master's thesis University College, Oxford University, (2002).

[30] P.W. Nelson, A.S. Perelson. Mathematical analysis of delay differential equation models of HIV-1 infection. Math. Biosci., 179 (2002), No. 1, 73-94. 
[31] S. Niculescu, P.S. Kim, D. Levy, P.P. Lee. On stability of a combined Gleevec and immune model of chronic myelogenous leukemia: exploiting delay system structure. Proceedings of 2007 IFAC Symposium on Nonlinear Control (2007).

[32] A. Papachristodoulou, M.M. Peet, S. Lall. Stability Analysis of Nonlinear Time-Delay Systems. IEEE Transactions on Automatic Control (Special Issue on Positive Polynomials in Control), 2009.

[33] P. Paschka, M.C. Muller, K. Merx, S. Kreil, C. Schoch, T. Lahaye, A. Weisser, A. Petzold, H. Konig, U. Berger, H. Gschaidmeier, R. Hehlmann, A. Hochhaus. Molecular monitoring of response to imatinib (Glivec) in CML patients pretreated with interferon alpha. Low levels of residual disease are associated with continuous remission. Leukemia, 17 (2003), No. 9, 1687-1694.

[34] M.M. Peet. Web site for Matthew M. Peet. http: //mmae.iit.edu/mpeet, (2009).

[35] M.M. Peet, A. Papachristodoulou, S. Lall. Positive forms and stability of linear time-delay systems. SIAM Journal on Control and Optimization, 47 (2009), No. 6, 3237-3258.

[36] A.S. Perelson, G. Weisbuch. Immunology for Physicists Rev. Mod. Phys., 69 (1997), No. 4, 1219-1267.

[37] L. Pujo-Menjouet, M.C. Mackey. Contribution to the study of periodic chronic myelogenous leukemia. Comptes Rendus Biologiques, 327 (2004), 235-244.

[38] I. Roeder, M. Horn, I. Glauche, A. Hochhaus, M.C. Mueller, M. Loeffler. Dynamic modeling of imatinib-treated chronic myeloid leukemia: functional insights and clinical implications. Nat. Med., 12 (2006), No. 10, 1181-1184.

[39] C.L. Sawyers. Chronic myeloid leukemia. New Engl. J. Med., 340 (1999), No. 17, 13301340.

[40] C.L. Sawyers, A. Hochhaus, E. Feldman, J.M. Goldman, C.B. Miller, O.G. Ottmann, C.A. Schiffer, M. Talpaz, F. Guilhot, M.W. Deininger, T. Fischer, S.G. O'Brien, R.M. Stone, C.B. Gambacorti-Passerini, N.H. Russell, J.J. Reiffers, T.C. Shea, B. Chapuis, S. Coutre, S. Tura, E. Morra, R.A. Larson, A. Saven, C. Peschel, A. Gratwohl, F. Mandelli, M. BenAm, I. Gathmann, R. Capdeville, R.L. Paquette, B.J. Druker", Imatinib induces hematologic and cytogenetic responses in patients with chronic myelogenous leukemia in myeloid blast crisis: results of a phase II study. Blood, 99 (2002), No. 10, 3530-3539.

[41] C.A. Schiffer, R. Hehlmann, R. Larson. Perspectives on the treatment of chronic phase and advanced phase CML and Philadelphia chromosome positive ALL. Leukemia, 17 (2003), No. 4, 691-699.

[42] G. Stengle. A nullstellensatz and a positivstellensatz in semialgebraic geometry. Mathematische Annalen, 207 (1973), 87-97. 
[43] J.F. Sturm. Using SeDuMi 1.02, a Matlab toolbox for optimization over symmetric cones. Optimization Methods and Software, (1999), vol. 11-12, 625-653, Version 1.05 available at http:/ / fewcal.kub.nl/sturm/software/sedumi.html.

[44] S.F.T. Thijsen, G.J. Schuurhuis, J.W. van Oostveen, G.J. Ossenkoppele. Chronic mlyeloid leukemia from basics to bedside. Leukemia, 13 (1999), No. 11, 1646-1674.

[45] M. Uzunel, J. Mattsson, M. Brune, J-E. Johansson, J. Aschan, O. Ringden. Kinetics of minimal residual disease and chimerism in patients with chronic myeloid leukemia after nonmyeloablative conditioning and allogeneic stem cell transplantation. Blood, 101 (2003), No. 2, 469-472.

[46] M.J. van Stipdonk, E.E. Lemmens, S.P. Schoenberger. Nä̈ve CTLs require a single brief period of antigenic stimulation for clonal expansion and differentiation. Nat. Immunol., 2 (2001), No. 5, 423-429.

[47] M. Villasana, A. Radunskaya. A delay differential equation model for tumor growth. J. Math. Biol., 47 (2003), No. 3, 270-294. 